\title{
Periods in Solar Activity
}

\author{
Amy L. Potrzeba-Macrina ${ }^{1}$, Igor G. Zurbenko² \\ ${ }^{1}$ Syracuse University, Department of Mathematics, 215 Carnegie Building, Syracuse, NY 13244, USA \\ ${ }^{2}$ University at Albany, Department of Epidemiology \& Biostatistics, 1 University Place, Rensselaer, NY 12144, USA \\ Email: amacrina@syr.edu
}

\begin{abstract}
Solar activity has a well-known periodicity of approximately 10-11 years, an oscillation that was first observed in China several thousand years ago. The purpose of this paper is to explain the driving force behind this periodicity and to explain other periodicities inherent to solar activities. In science, spectral analysis is an essential tool used for the identification of periodicities that are natural to a given dataset. In this paper the authors use spectral analysis to investigate planetary gravitational periods to explain periodicities of sunspot numbers and to make conclusions about the driving force of the sunspot numbers and solar activity. Precise analysis of inherent periodicities provides the capability to predict future fluctuations in solar activities. The authors show clear evidence of long periodicities within sunspot numbers. The combination of several periodic components, while complex, remains perfectly predictable. The authors show that the long-term component of sunspot fluctuations is perfectly proportional to the total solar irradiation near Earth measured by satellites. While satellite measurements of the total solar irradiance cover a short time interval, sunspot numbers have been recorded for a long time and essentially have more value on the prediction of solar influence on Earth's climate. This allows for the numerical evaluation of solar energy delivered to Earth. Numerical evaluations of fluctuations in solar energies delivered to Earth are an essential achievement for any climate change analysis. The removal of solar influences from long-term temperature data provides the opportunity to numerically identify the human impact on Earth's climate. A better understanding and prediction of the Sun's long oscillations may influence important predictions of climatic events and impact emergency preparedness.
\end{abstract}

Keywords: difference frequency, spectrum of sunspots, planet periods, extreme weather events, solar radiation

\section{Introduction}

Historically, there have been fluctuations in solar activities. A periodicity of approximately 10-11 years was observed in average sunspot numbers and has been a research focus for many researchers [1]. However, there has yet to be a clear explanation of the very precisely organized periodicities found in sunspot numbers.

Sunspot numbers and their effects are the topic of a variety of research. For example, Ahluwalia [2] attempts to predict future solar cycle activity by using some long periods in sunspot numbers but does not explain the source of the long periods nor the exact value of the periods due an insufficient length of observations. Pesnell [3] makes a prediction of a high-level cycle in solar activity by determining the time lag with a geomagnetic precursor. Whereas, $\mathrm{Ng}$ [4] discusses the statistical estimation of time lag between magnetic polls orientation on the Sun and solar cycle and Marra and Morabito [5] approximate solar activity using the sum of sinusoids and apply this to Elman Networks which permits the forecasting of sunspot numbers. Similar to Marra and Morabito [5] the authors also approximate solar activity using the sum of sinusoids. However, the authors use spectral analysis to determine the sinusoids from the actual sunspot numbers. The authors of this paper consider the connection between the magnetic polls orientation on the Sun and the solar cycle as a joint mechanism of solar activity and concentrate on long-term sunspot investigations. In another research pertaining to sunspot numbers Chernykh [6] applies wavelet analysis to sunspot numbers by taking periods of some separate planets around the Sun, whereas the authors further consider the interactions between the planets' frequencies. Difference frequencies and multiple interaction frequencies provide several well-organized periods in solar 
activity. The authors' approach concentrates on frequency analysis and an explanation of strict frequency lines in the spectrum of sunspot numbers by period analysis in the total solar and planetary system. The authors consider this to be so well organized a frequency that most likely it is arising from a precise frequency in the planetary movements.

The Sun has 8 planets and their gravities provide disturbances to the Sun's mass. As the Moon creates tides on the Earth all planets must create their own tide on the Sun. The periodicity of such a tide will be equal to the time of rotation of the Sun around its axes extended by the shift in the sky of that planet during that single rotation. Different planets will provide different shifts due to their time of total rotation around the Sun. At times, some of those tides will be in-phase and other times in opposite phase which creates an oscillation of the amplitude of the tide created by two specific planets. This provides the well-known effect of a phase accumulation or difference frequency in oscillation of amplitude of the tide created by those two planets [7]. The authors calculate that the difference frequency of oscillation of amplitudes created by the largest planets, Jupiter and Saturn, is close to a period of 11 years.

The Moon creates tides on Earth strictly on the surface of the ocean under the Moon. Those tides will be shifted by the Earth's rotation in the eastern direction and its shift speed at the equator is 1000 miles per hour. The tidal wave shifted east will pool back by the Moon's gravity in the western direction. It is noticed that the equatorial current in all oceans is in the western direction. This same effect is observed in the atmosphere [8]. Tides on the Sun created by the planets will affect transmission of the mass in a direction that is opposite to the Sun's rotation. It is likely that plasma masses have very low friction for current transmission, which may create the effect known as a different speed of rotation of the Sun at its equator compared to further away from it. This speed ranges from an approximately 24.47 to 38 day period or 1 Sun Day (SD). Due to the influences of different planets' rotational speeds top layers sometimes exceed the core speed and sometimes are below it. This might result in the different magnetic polar orientation on the Sun noticed recently with a period of 11 years [9].

Different amplitudes of tides might create differences in speed. Differences in speed might create disturbances in the top layers of the Sun. Those disturbances contribute to the sunspots and electromagnetic explosions in the top layers with extra energies released from the highly active thermonuclear core of the Sun. That activity would have the same periods as tidal amplitudes of waves on the Sun. Since those periods are dictated by highly precise astronomic periods in the Sun's system, they will be very accurate in time.

Precise numerical observations of sunspot numbers have been available since the mid-1700s. Periodicities of approximately 8-11 years are perfectly observed in that collected data. The data also contains some longer periods, but those cannot be precisely detected due to the insufficient length of the data. According to Valachovic and Zurbenko [10] sunspot numbers are perfectly correlated with Earth's total solar radiation. Low solar activity in the early 1800s caused some climate problems in Europe. There have been some very long oscillations in solar activity resulting in dramatic variations of the Earth's climate. However, periodicities of those oscillations are not known due to lack of direct long measurements. The authors conduct a simulation with high computational capacity to simulate very long oscillations in solar activity. The simulations provide periods of 8-11 years in addition to some much longer periods. The periods of 8-11 years in the simulated model are in very good compliance with sunspot numbers allowing for the conclusion that the model is correct. The authors also investigate a relationship between total solar irradiance and sunspot numbers. The authors argue in section 6 that sunspot numbers can be used to estimate total solar irradiance which provides the opportunity to further investigate long-term oscillations of total solar irradiance and its effects on the climate.

\section{Data Sources}

For the analyses in this paper the authors consider average monthly sunspot data, total solar irradiance (TSI) data and temperature data at a tropical location. The authors retrieved average monthly sunspot data online from the Sunspot Index and Long-term Solar Observations (SILSO) [11]. For the TSI data, the authors retrieved the dataset, which contained daily TSI measurements in watts per meter squared from the Active Cavity Radiometer Irradiance Monitor (ACRIM). This data is free and available at the website: http://acrim.com/ and the authors downloaded file was Virgo version 6_005_1602 created on 
March 14, 2016 [12]. The TSI dataset included measurements from January 1996 to present [12], but the authors only considered the data values between the years 2000-2015 due to the missing values in the beginning years of the dataset. The authors also downloaded and accessed subsets of average monthly temperature data from tropical regions that were collected by the Research Data Archive/Computational and Information Systems Laboratory/National Center for Atmospheric Research/University Corporation for Atmospheric Research in $2^{\circ}$ by $2^{\circ}$ grids [13]. For their analyses the authors used subsets of the data ds540.1, the International Comprehensive Ocean-Atmosphere Data Set (ICOADS) [13].

\section{Observation of Solar Periods in Sunspot Data}

Sunspot numbers were observed in China more than 2000 years ago with the first sighting dating back to $28 \mathrm{BC}$ [14]. More accurate observations were made with telescopes in the 1600s and have been recorded since the 1700s [1]. The SILSO dataset [11] contained monthly mean total sunspot numbers dating back to January 1749. The authors used this data (see Figure 1) to determine the spectrum of the data and then used the Kolmogorov-Zurbenko filter package in R-software to smooth the data using the DiRienzo-Zurbenko (DZ) smoothing method [15], [16].

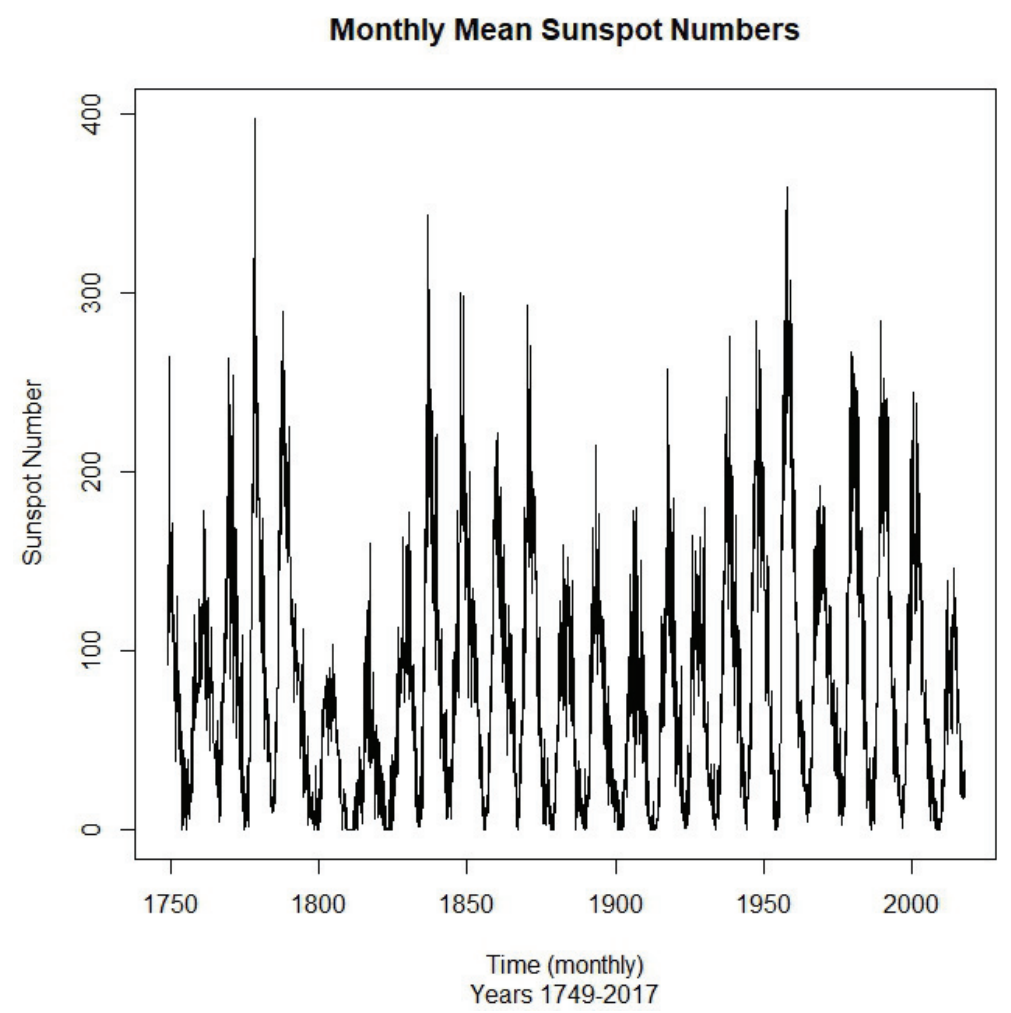

Figure 1. The graph depicts monthly mean sunspot numbers from 1/1749 - 8/2017.

Regular statistical estimations of spectra use a fixed spectral window smoothing [17]. Unfortunately, this use of a fixed spectral window frequently smooths out some of the spectral lines. The DZ algorithm addresses actual values of the spectrum in order to select the width of smoothing with a given percentage of total energy. The width of smoothing depends on actual values of the spectrum so that it perfectly captures any concentrations of energy above the selected percentage number and identifies their locations. If there are separate spectral lines exceeding the selected threshold then the energy of it will be displayed as separate lines. Obviously the percentage number should be above $1 / \mathrm{N}$ where $\mathrm{N}$ is the sample size. It is the choice of the researcher to choose the percentage number. Thus the algorithm smooths energies below the set percentage and returns locations of the energies above it. DiRienzo \& 
Zurbenko [16] use real and simulated examples to demonstrate accuracy of the determination of separate spectral lines using the DZ algorithm and discuss the algorithm's advantages over using regular spectral windows for smoothing. Computer codes of the DZ algorithm are provided in the KZA package in Rsoftware [18].

As mentioned in the previous paragraph the DZ smoothing method requires a parameter that is the percentage of total variation. For the sunspot data, a low percentage (1\%) resulted in a spectrum that not only identified the known periods of approximately 8-11 years, but also a long oscillation of approximately 90 years. The authors determined there were not enough data points to make conclusive results about the value of long periodicities. However, one will see through the authors' simulated study (described in section 5) that solar activity has very long oscillations. Furthermore, long-term oscillations in sunspot numbers have been observed for example [1] discusses solar cycles with 90-100 year and 50-60 year periods. More raw data is needed to draw substantial conclusions pertaining to the long oscillations.

When smoothing the spectrum with the DZ algorithm the authors chose a percentage of $20 \%$ and the result (see Figure 2) identified three main peaks. Two periods of 10.4 and 8.7 years were identified and are sufficiently close to the true periods and the third peak corresponded to a period of approximately 19.3 years. The longer period demonstrates the presence of long periods in solar activity. Using the information from the smoothed spectrum the authors decomposed the raw data into the sum of its longterm component, mid-scaled component (approximately 8-11 year scale) and residuals (short-term component). The authors used the Kolmogorov-Zurbenko, $(\mathrm{KZ}(\mathrm{m}, \mathrm{k}))$, filter to extract the long-term component from the raw data. The $\mathrm{KZ}$ filter is in the KZA package of $\mathrm{R}$-software and requires two parameters: window size $(\mathrm{m})$ and number of iterations $(\mathrm{k})$ [18]. The KZ filter is an iterated moving average; for more information on KZ-filtration and its advantages refer to Yang \& Zurbenko [19], Zurbenko \& Smith [20] and Valachovic \& Zurbenko [21]. The authors extracted the long term component by applying KZ(10.4 years, 3) to the raw sunspot data (see Figure 3).

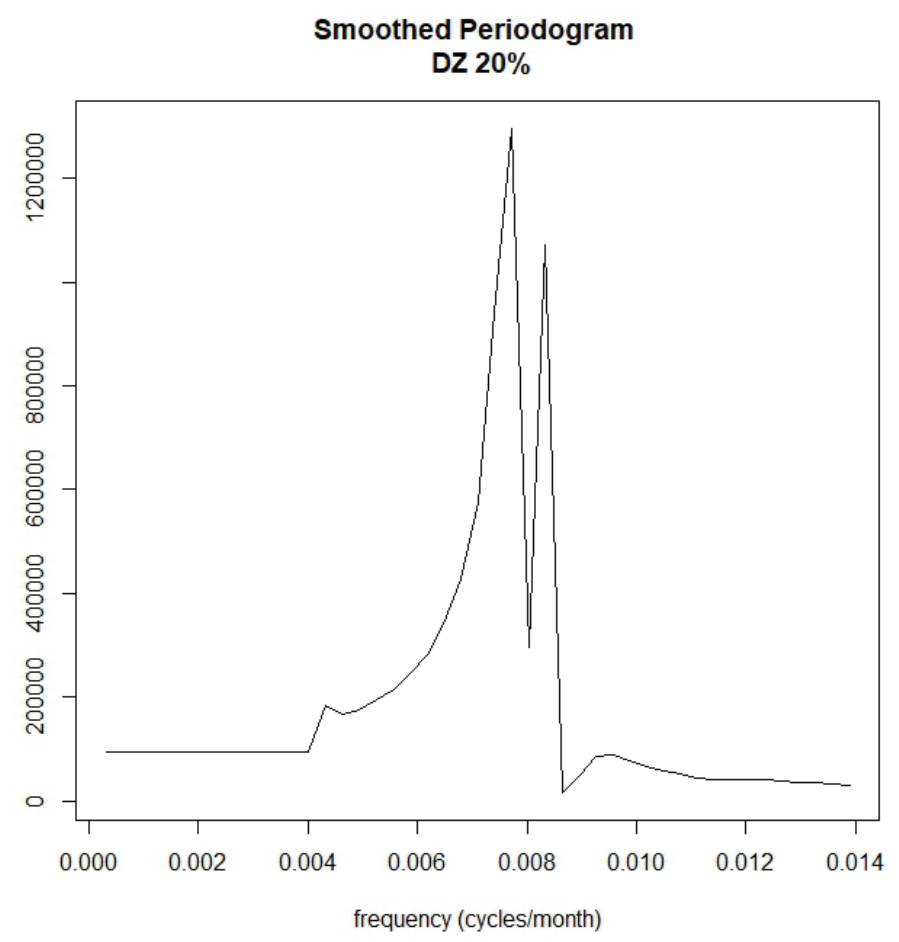

Figure 2. The smoothed spectrum of the Mean Monthly Sunspot data using the DZ algorithm with a percentage of total variation equal to $20 \%$. The main peaks correspond to periods of 19.3 years, 10.4 years, and 8.7 years. 


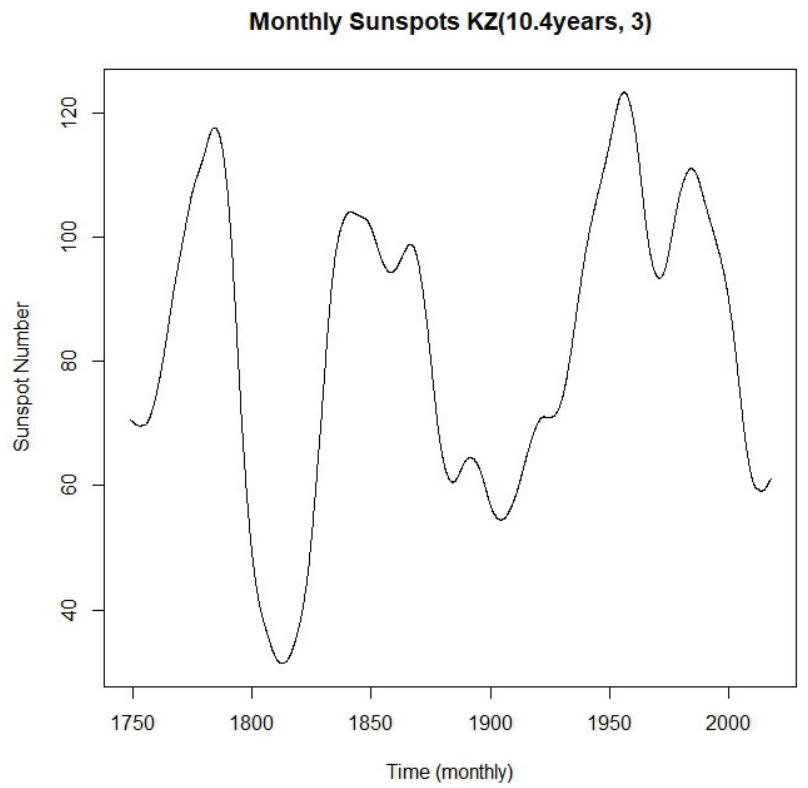

Figure 3. Long term component of the raw sunspot data using KZ(10.4 years, 3).

The authors reconstructed the 8-11 year mid-scaled component of the sunspot data (see Figure 4) by applying the filter $\mathrm{KZ}(1$ year, 3) to the raw data less the long-term component and then calculated the short-term component or residuals (see Figure 5). A QQ-normal plot of the residuals with the linear regression line (Figure 6) was analyzed. The $\mathrm{R}^{2}$ value is $95 \%$ meaning it is a good fit to the normal distribution with a mean (intercept value) of -0.01338 , which is indistinguishable from 0 . The standard deviation (slope) is 22.28243. These results confirm that the residuals (or short-term component) are normal noise and therefore the long-term and 8-11 year mid-scaled components as obtained by the authors are accurate. Compared to the early 1900s both the long-term and mid-scaled (8-11 year) components are in a low phase. The Earth's atmospheric temperature values typically are delayed compared with the solar energy source.



Figure 4. Scaled Component of the Sunspot Data. KZ (1 year, 3) applied to the raw data less the long term component. 
Residuals= Raw Sunspot - Long Term Component - Scale



Figure 5. Plot of the residuals determined by removing the long term and 8-11 year scaled components from the raw sunspot data.

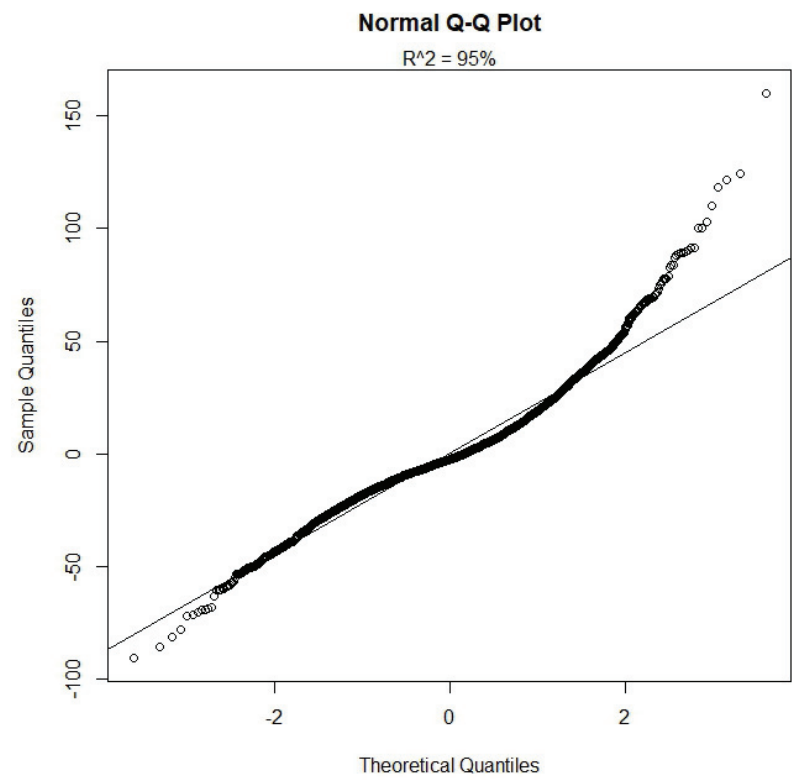

Figure 6. QQ - Normal plot of the residuals with the linear regression line. $\mathrm{R}^{2}$ value is approximately $95 \%$.

\section{Difference Frequency}

It has been observed that the sunspots have two periodicities in the range $8-11$ years as discussed in literature including Valachovic \& Zurbenko [10] and Zurbenko \& Potrzeba [7]. Periodic signals are natural to many atmospheric variables. Zurbenko and Potrzeba [7] discuss periodic signal reconstruction and the concept of difference frequencies, in particular, they discuss this phase accumulation with atmospheric pressure. A difference frequency (or phase accumulation) is the fundamental concept that if two different waves are in-phase once they will also be out-of-phase for a time which generates an oscillation of the amplitude of the sum of those waves.

As Zurbenko and Potrzeba [7] discuss the phase accumulation caused by the Moon on the Earth, this may also be similarly repeated for the Sun. The Sun has lots of planets that all provide tides with specific periodicities that will accelerate with a difference frequency. In this section the authors discuss 
the tides caused by the planets with the main contributors being Jupiter and Saturn, but all of the planets' tides contribute to the phase accumulation and the 8-11 year periodicities.

The authors argue that the 8-11 year period can be the result of a difference frequency. There are 8 planets whose tides are acting on the Sun. These tides are harmonic oscillations that when considering the total sum provide a complex signal that results in amplitude modulation of a low difference frequency. The joint effect of gravity of all the planets over the Sun's semidiurnal periods may explain long periods in the solar activity and partially explain climate fluctuations over Earth [22].

The Sun turns around its own axis at the equator in 24.47 days (1 Sun Day = SD). The largest planet in the Sun's system is Jupiter, which is rotating around the Sun in 4332.82 days. During 1 SD, Jupiter loses one SD so it is appearing over the same spot on the Sun in 24.608 days. The second largest planet is Saturn and it appears over the same spot on the Sun in 24.526 days. The periods of rotation for each of the planets appearing over the same coordinate location on the Sun are summarized in Table 1. While the accuracy of the speed of the Sun's rotation should be investigated more precisely it minimally affects the difference frequency which the authors are using for their conclusions. The difference frequency depends more on the periods of the planets around the Sun, which are precisely known.

Table 1. Information about each of the 8 planets in the Sun's system.

\begin{tabular}{cccccccc}
\hline Planet & $\begin{array}{c}\text { Distance from } \\
\text { the Sun (AU) }\end{array}$ & $\begin{array}{c}\text { Earth } \\
\text { masses }\end{array}$ & $\begin{array}{c}\text { Period } \\
\text { (days) }\end{array}$ & $\begin{array}{c}\text { Period } \\
\text { (years) }\end{array}$ & $\mathbf{m} / \mathbf{r}^{2}$ & $\begin{array}{l}\text { Period (in days accounting } \\
\text { for Sun rotation) }\end{array}$ & $\begin{array}{l}\text { Semi Periods } \\
\text { (days) }\end{array}$ \\
\hline Mercury & 0.387 & 0.055 & 87.97 & 0.24 & 0.367 & 31.277 & 15.638 \\
Venus & 0.723 & 0.815 & 224.7 & 0.615 & 1.559 & 27.135 & 13.567 \\
Earth & 1 & 1 & 365.26 & 1 & 1.000 & 26.109 & 13.055 \\
Mars & 1.52 & 0.107 & 686.98 & 1.881 & 0.046 & 25.342 & 12.671 \\
Jupiter & 5.2 & 317.8 & 4332.8 & 11.86 & 11.753 & 24.608 & 12.304 \\
Saturn & 9.54 & 95.2 & 10756 & 29.46 & 1.046 & 24.526 & 12.263 \\
Uranus & 19.2 & 14.5 & 30687 & 84.01 & 0.039 & 24.490 & 12.245 \\
Neptune & 30.1 & 17.1 & 60190 & 164.8 & 0.019 & 24.480 & 12.240 \\
\hline
\end{tabular}

A single "moon" with an orbit that is close to circular provides a sufficiently stable tide. A pair of planets each provides a tide, but periods of those tides appear differently due to the differing speeds of each planet on the horizon. The two different oscillations are at one time in-phase and then later in opposite phases. This amplitude modulation has a period that is a difference frequency (or phase accumulation) of the two original oscillations [7]. As an example, the authors consider the two planets Jupiter and Saturn. The semi-frequencies of Jupiter and Saturn over the Sun are approximately 0.081271 and 0.0815468 cycles per day; respectively. Therefore, the difference frequency of Jupiter and Saturn appearing over the Sun is equal to 0.0002758 cycles per day which corresponds to a period of 9.933742 years. In the same way, the authors calculate the difference frequency separately of the tides created by Venus and Earth. Their semi-frequencies are 0.073708 and 0.076599 cycles per day, respectively. Therefore, the difference frequency of Venus and Earth is 0.002891 cycles/day which corresponds to a period of approximately 0.947 years. Venus and Earth provide a short period compared to the one created by Jupiter and Saturn. Approximately every 8.6, 9.9 and 10.5 years, Venus, Earth and the Sun are approximately in line making the strongest joint gravitational effect on a bimodal tidal wave over the Sun. For two planets the difference frequency of the tides does not depend on the amplitudes of the tides caused by each planet. If there are more than two planets simultaneously the concept becomes more complex and depends on the amplitude of each tide. Jupiter and Saturn provide a long period and high amplitude oscillation, whereas Venus and Earth provide a short period and low amplitude oscillation. The interference of these oscillations may cause small shifts of the strong oscillation caused by Jupiter and Saturn. The authors demonstrate this by composing those two oscillations together and determining the raw spectrum of this composition (see Figure 7). This simulation serves only as a clue of a secondary peak in the spectrum caused by the joint effect of the strong gravities of Jupiter and Saturn and is not a precise calculation. In order to fully understand the total effect of all oscillations it would be necessary to conduct a total simulation of all planetary tides. Since the total effect of the 8 planets cannot be calculated theoretically the authors conducted a simulated study of it that is detailed in the next section. 


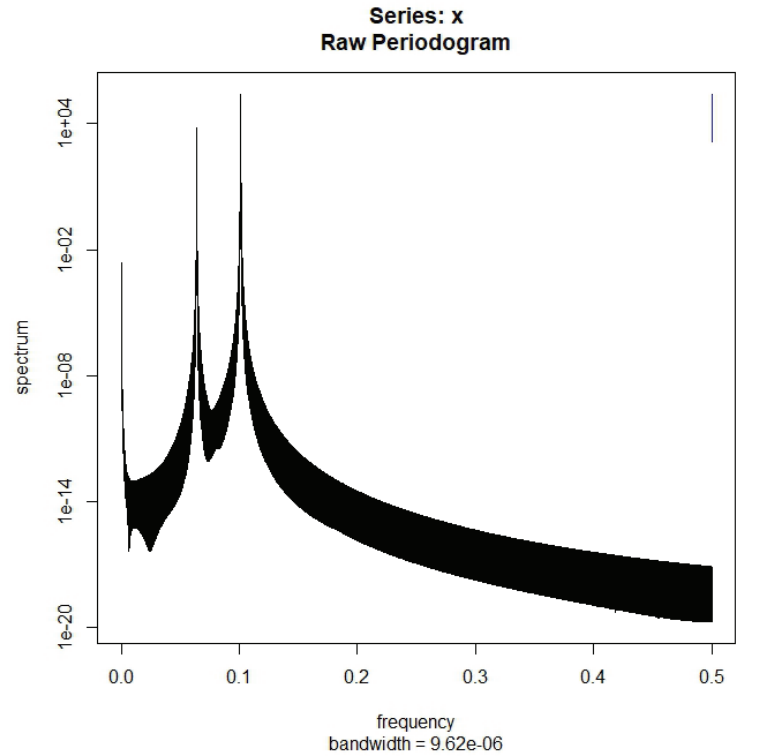

Figure 7. The spectrum of the simulated signal $y(t)=7 \sin \left(2 \pi\left(\frac{1}{9.9}\right) t\right)+\sin \left(2 \pi\left(\frac{1}{0.94}\right) t\right)$. The frequencies are displayed is cycles/year.
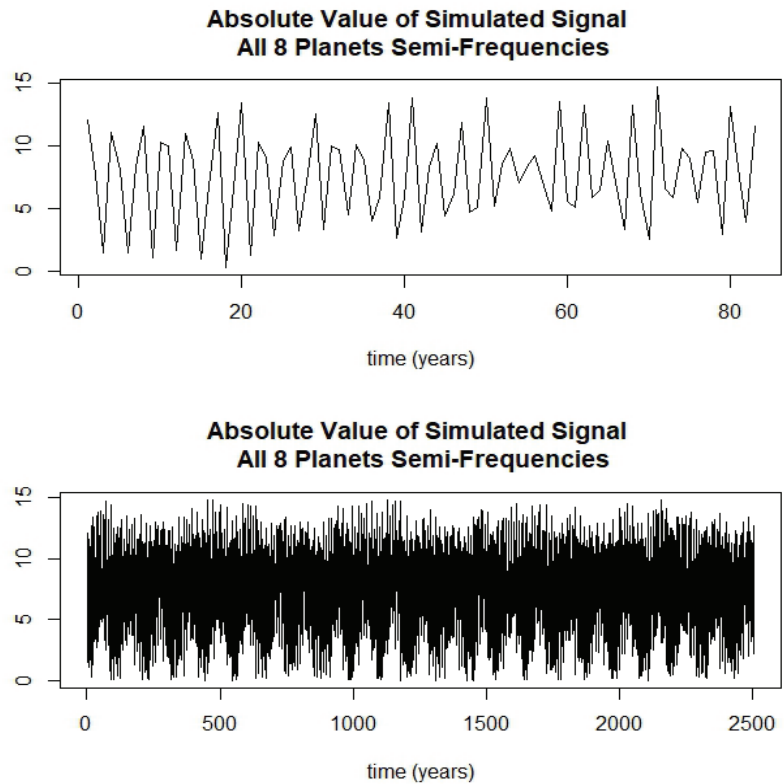

Figure 8. Two plots of the absolute value of the simulated signal using the semi-frequencies of all 8 planets. The absolute value is considered in order to investigate low frequencies in the spectrum. The top plot shows the signal over approximately 80 years and the bottom plot shows the signal extended to approximately 2500 years.

\section{Simulated Study}

The purpose of this section is to simulate a signal that emulates the total effect of all 8 planets to investigate the claim that the 8-11 year solar period is the result of a difference frequency in addition to comparing any long-term oscillations in the simulation to those determined from the smoothed spectrum of the sunspot data. The authors constructed a signal that is the sum of eight sine waves where each wave has a frequency that corresponds to the semi-frequency of one of the 8 planets in the solar system. The authors chose the amplitude of each sine wave to be the mass of the corresponding planet (in Earth 
masses) divided by the square of the distance between the planet and the Sun. The reasoning for this seclection is that according to Newton's law of gravity, the gravitational force is equal to GMm $/ \mathrm{r}^{2}$ and Newton's constant, G, and mass of the Sun are the same for each. The planetary information is summarized in Table 1. Since the authors wanted to investigate low frequencies in the spectrum they then took the absolute value of this signal (Figure 8).

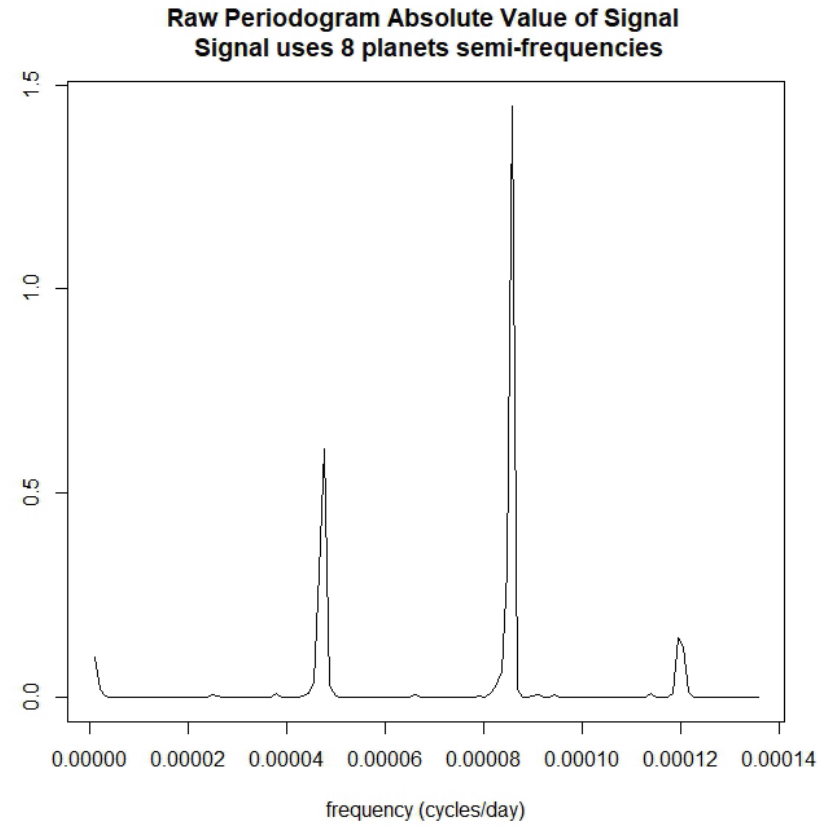

Figure 9. The spectrum of the absolute value of the simulated signal after using KZ (2 months, 3). The peaks correspond to periods of 57.3 years, 31.9 years, and 22.9 years. The elevation near 0 is evidence of a long period in sunspots.



Figure 10. The spectrum of the absolute value of the simulated signal after using KZ (2 months, 3). The main peak corresponds to a period of approximately 10.1 years. 


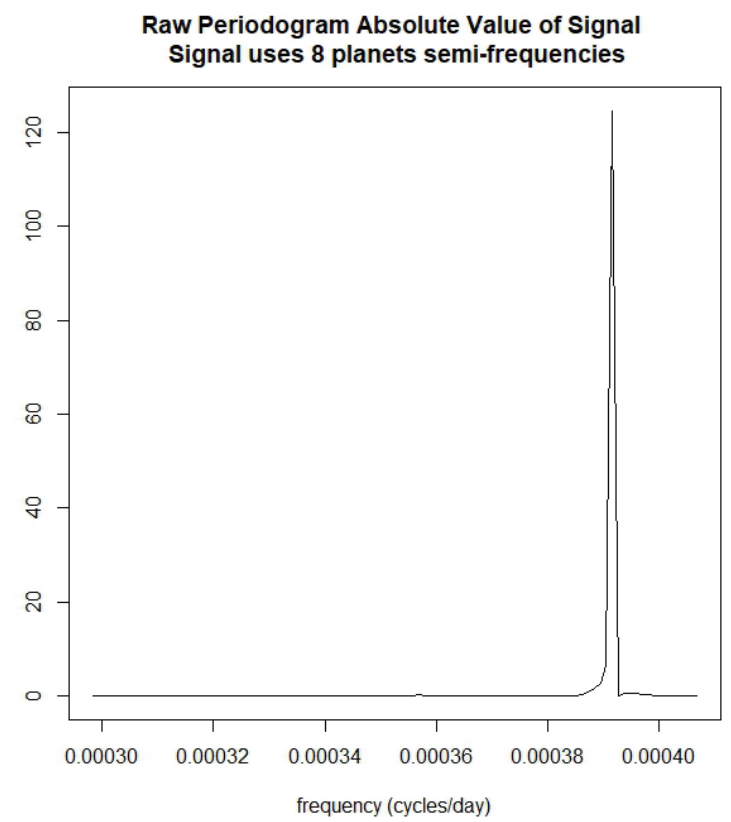

Figure 11. The spectrum of the absolute value of the simulated signal after using KZ (2 months, 3). The main peak corresponds to a period of approximately 7 years.

The signal which is also viewed in monthly time units provides the same information as the real sunspot data with the advantage that it can be used to predict and analyze very long oscillations in solar energy since it allows a longer dataset. This opportunity is not available for the raw sunspot data since one is limited by the length of the available sunspot data. The authors smoothed the simulated signal by applying KZ(2 months, 3). The spectrum of this smoothed signal (Figures 9, 10 and 11) revealed main peaks corresponding to frequencies of approximately 58, 32, 23, 10 and 7 years.

Table 2. Period comparisons between spectra of sunspot data and simulations.

\begin{tabular}{|c|c|c|c|c|c|}
\hline \multicolumn{2}{|c|}{ Monthly Sunspot Data } & \multicolumn{2}{|c|}{ Monthly Sunspot Data } & \multicolumn{2}{|c|}{ Simulation KZ(2 months, 3$)$} \\
\hline \multicolumn{2}{|c|}{ DZ $1 \%$ level } & \multicolumn{2}{|c|}{ DZ $20 \%$} & \multicolumn{2}{|c|}{ Absolute Value $\mathrm{KZ}(2$ months, 3$)$} \\
\hline $\begin{array}{c}\text { Frequency } \\
\text { (cycles/month) }\end{array}$ & $\begin{array}{l}\text { Period } \\
\text { (years) }\end{array}$ & $\begin{array}{c}\text { Frequency } \\
\text { (cycles/month) }\end{array}$ & $\begin{array}{l}\text { Period } \\
\text { (years) }\end{array}$ & $\begin{array}{c}\text { Frequency } \\
\text { (cycles/day) }\end{array}$ & $\begin{array}{l}\text { Period } \\
\text { (years) }\end{array}$ \\
\hline 0.000925926 & 90.0000025 & 0.00432099 & 19.28571274 & 4.77431E-05 & 57.34552388 \\
\hline 0.007716049 & 10.8 & 0.00802469 & 10.38461585 & $8.57205 \mathrm{E}-05$ & 31.93928064 \\
\hline 0.008333333 & 10 & 0.00957901 & 8.699576818 & 0.000119358 & 22.93821916 \\
\hline 0.009876543 & 8.43750001 & & & 0.000271267 & 10.09281169 \\
\hline & & & & 0.00039171 & 6.98948224 \\
\hline
\end{tabular}

The authors compared the results from the simulation to the periodicities determined from the spectrum of the smoothed sunspot data. The results are summarized in Table 2. The simulations support the authors' claim that the 8-11 year period must be occurring naturally as difference frequency (as was calculated in the previous section). The authors argue that the long oscillation of approximately 57 years also is the result of a difference frequency between the periods of approximately 10.4 years and 8.7 years. A period of 10.4 years is approximately 125 months, which provides a frequency of $1 / 125=$ 0.008 cycles/month. A period of 8.7 years is approximately 105 months, which provides a frequency of $1 / 105=0.0095238$ cycles $/$ month. The difference frequency is $0.0095238-0.008=0.0015238 \mathrm{cycles} / \mathrm{month}$ which is approximately 656.25 months or 54.7 years. An oscillation of $55+$ years is noticeable in the long term component of the sunspots (Figure 3). The knowledge of these long oscillations in solar activity may provide important knowledge that could be useful in predicting climatic events as well as supporting emergency preparedness efforts for such events. The long-term component clearly was in decline in the last decade and the mid-range, scaled component of 8-11 years, was also in decline 
(Figures 3 and 4), which yields very low solar activity. Zurbenko and Cyr [22] notice that sunspots' long-term oscillations are correlated with annual temperatures, which in turn may cause problems similar to those in the early 1800s with "no summer in Europe."

\section{Solar Irradiation Fluctuations}

The Oxford dictionary of Astronomy defines total solar irradiance (TSI) as the measure of the radiation flux from the Sun received by Earth over all wavelengths. The purpose of this section was to determine if there was a relationship between sunspot numbers and TSI. Since direct correlation analysis can provide false results it was necessary to first remove the high frequency component in order to allow for an accurate analysis within low frequencies [23], [24]. After applying the KZ filter to remove stochastic short term fluctuations to both the TSI and sunspot numbers datasets, the authors determined that there was a linear relationship between the two datasets, with an $R^{2}>98 \%$. However, due to the length of the dataset and the KZ filter's design [19], there was an edge effect to this result. Namely, as the filter got close to the edge it enhanced the effects of prior data observations. Since the available TSI dataset was short it could not be extended longer to reapply the filter to avoid such an effect. However, due to the strength of the linear relationship between sunspot numbers and TSI the authors were able to correct the edge effect. In order to extend the TSI dataset, which is to say predict it beyond the available timeframe, the authors used the linear regression model from the first application to predict the TSI from the sunspot numbers. Using these predicted TSI values to extend the TSI dataset, the KZ filter was reapplied to extended TSI dataset and compared with the smoothed sunspot numbers data. The result showed a higher correlation-squared value; $R^{2}>99 \%$ (see Figure 12). Therefore, long-term or baseline components are highly correlated after removing the short-term (or higher frequency) fluctuations, thus leaving no doubt that there is a linear relationship between long-term components of TSI and sunspot numbers.

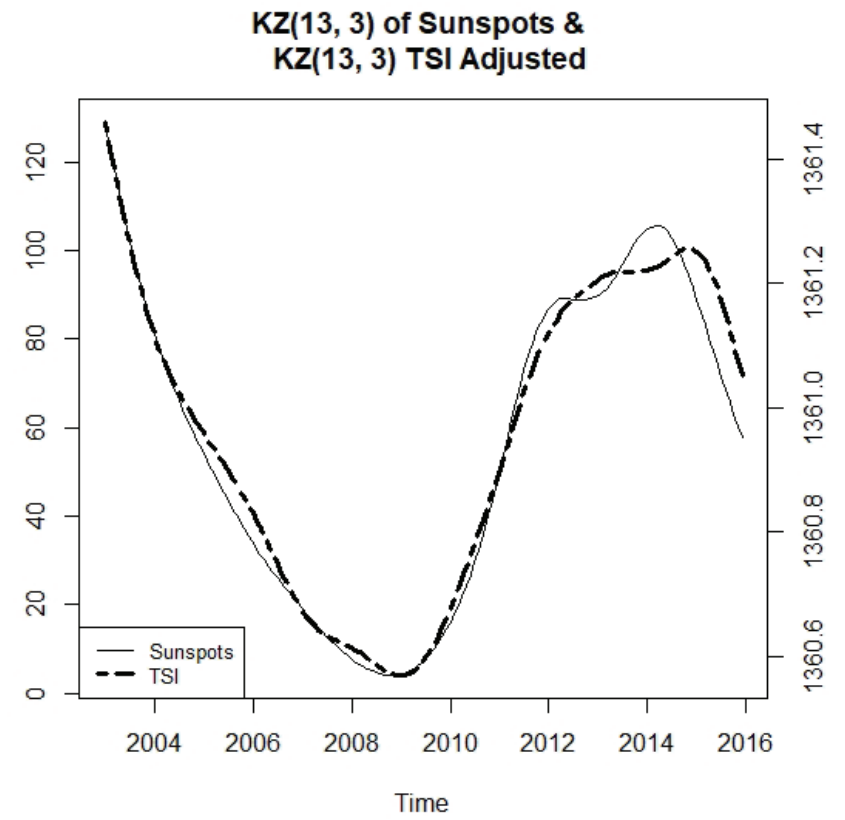

Figure 12. The plot shows the results of the smoothing filter, KZ(13 months, 3) applied to both sunspot numbers and to the extended TSI data. The TSI measurements are on the right vertical axis and sunspot numbers on the left vertical axis. The dashed line is the KZ(13 months,3) of TSI whereas the solid line represents $\mathrm{KZ}(13,3)$ of sunspot numbers. The horizontal axis is time in months. $R^{2}>99 \%$. 


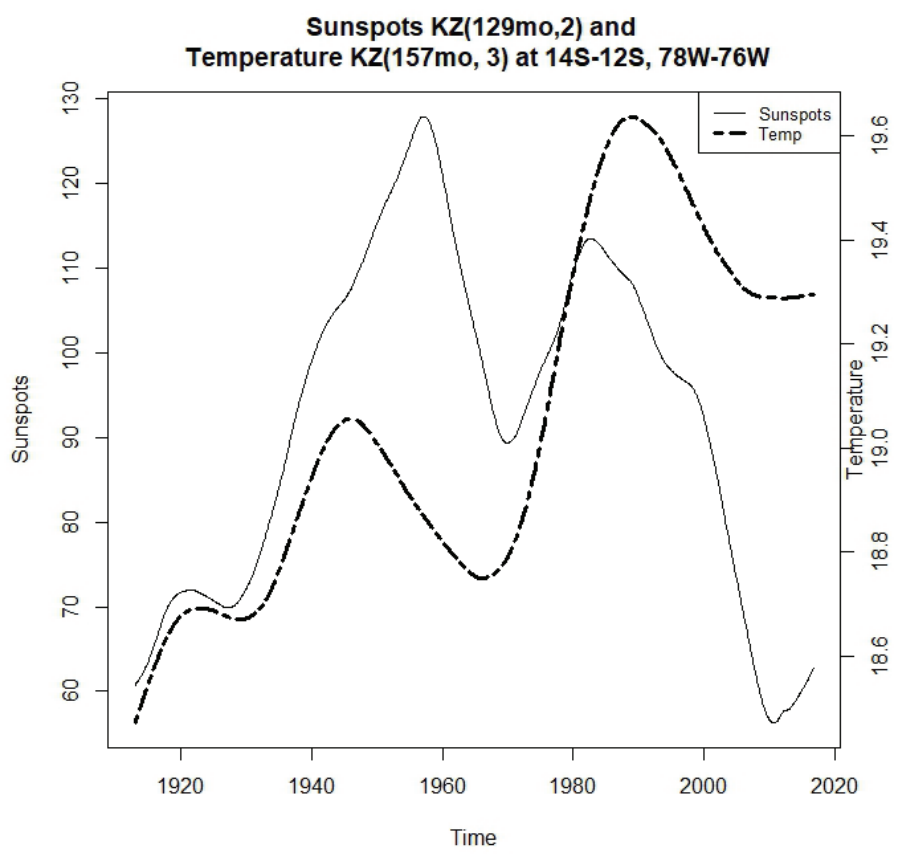

Figure 13. The graph displays the results of KZ(129 months, 2) to sunspot numbers in a solid line and $\mathrm{KZ}(157$ months, 3) in temperature in a $2^{\circ} \times 2^{\circ}$ grid in Peru in a dashed line. The left side vertical axis depicts sunspot numbers and the right vertical axis depicts temperature. The horizontal axis is time in months.

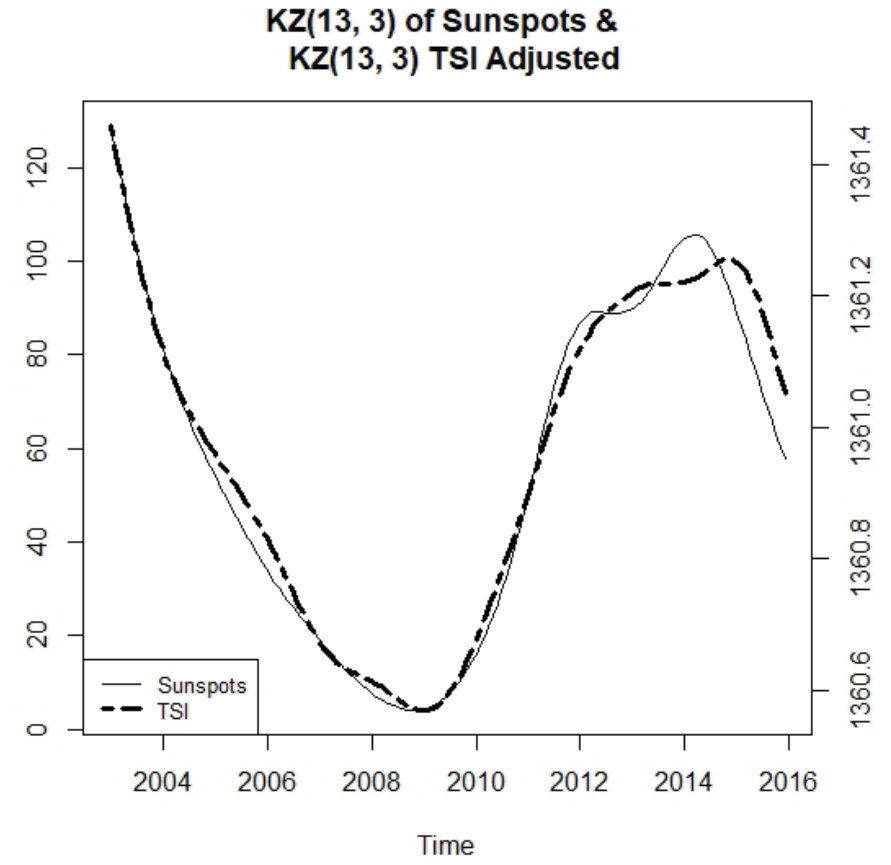

Figure 14. Unexplained portion of solar radiation within temperature data in Peru.

Due to the strong linear relationship between sunspot numbers and TSI sunspot numbers can be used to estimate TSI or fluctuations in solar radiation near Earth. Since this was possible, the authors were able to use these estimations in order to further investigate long-term oscillations and climate effects. In 
order to investigate long-term temperature oscillations alongside long-term oscillations in sunspot numbers, temperature data at Peru was used for this investigation because Peru is a tropical location near the equator. The authors first removed periods close to 10 years in sunspot numbers by applying the KZ filter with a window size of approximately 10.8 years (129 months) and removed periods close to 13 years in temperature by applying the KZ filter with a window size of 13.1 years (157 months) to temperature data collected at Peru (see Figure 13). It was evident that the oscillations in both datasets are similar with a correlation of 0.42 . This result corresponds to Zurbenko and Cyr's [22] research which concluded a correlation of 0.56 over a time span of 250 years. The similar yet greater oscillations in the temperature dataset were then removed using a linear regression of the long-term temperature with the long-term sunspot numbers. From the previous discussion, one may note that the long-term sunspot numbers may also be considered as TSI. Using the linear regression the authors were able to remove the effect of TSI on the temperature data leaving the remaining unexplained portion (see Figure 14).

The result is that the unexplained portion of the solar irradiation fluctuations stems from human influence on the transfer of solar energy to Earth. It is also essential to note that there may also be regional differences, however, it is clear that solar irradiation fluctuations are in essence affecting extreme weather events. Zurbenko and Sun [25], [26], [27], provided undoubtable evidence of an increase in the frequency of tornados with higher solar irradiations. In addition, Zurbenko and Potrzeba [7], [8], [28] provide evidence of an increase in wind speeds of hurricanes with a higher solar 24-hour period irradiation.

\section{Conclusion}

It is evident to scientists that solar activity contains periodicities of 8-11 years. Accurate investigation of sunspot numbers reveal two spectral (frequency) lines corresponding to periods of approximately 8.7 and 10.4 years. The authors' investigation of the periodicities inherent to the Sun through simulation are accurate because they are based on gravitational forces caused by planets in the solar system and the plots of the simulation compared to the sunspot data support this. The periodicities in those forces are precisely organized, contain some periods within the 8-11 year range and provide some longer periods as well. Precise measurements of sunspot numbers are available only for approximately the past 268 years, but the planetary movements are easily calculable for thousands of years past and future. Hence, computer calculations permit the reproduction of positions and gravitational periods on the Sun for thousands of years. This reproduction reveals periods which are very close to the 8-11 year periods in sunspot numbers. Everywhere in science spectral lines are used as "fingerprints" to provide the identification of inherent natural evidences, which in this case permits the authors to make conclusions about the driving force of sunspot numbers and solar activity as planetary gravitational periodicities. Planetary systems have very well organized periods, which have been identified through spectral analysis. While long periods in sunspot numbers cannot be identified precisely due to the small sample size of observations, evidence of longer periods within sunspot numbers is undeniable. Through simulation, the authors demonstrate long oscillations that are naturally occurring in solar activity. Through simulation long periods can precisely be determined. This knowledge can provide key factors in the analysis of climate change and predictions. Solar activity is an important driver of the Earth's climate and weather. Therefore, by understanding the periods of the Sun one is able to more accurately predict climatic events on Earth.

\section{References}

1. Hathaway, D (2015). "The Solar Cycle." Living Reviews in Solar Physics. 12 (4). DOI: 10.1007/lrsp-2015-4

2. Ahluwalia, HS (2017). "Heuristic study of evolution of sunspot number timeline for next several cycles." $J$. Atmos. Solar-Terr. Phys. DOI:10.1016/j.jastp.2017.05.007. https://www.sciencedirect.com/science/article/pii/ S1364682616302449

3. Pesnell, W.D. (2014). "Predicting Solar Cycle 24 Using a Geomagnetic Precursor Pair." Solar Physics (ISSN 0038-0938). 289 (6), p. 2317-2331. DOI 10.1007/s11207-013-0470-x

4. Ng, K. Kwee (2016). "Prediction Methods in Solar Sunspots Cycles." Scientific Reports (www.nature.com/ scientificreports/) DOI: 10.1038/srep21028 
5. Marra S, \& Morabito, F (2005). "A New Technique for Solar Activity Forecasting using Recurrent Elman Networks." International Journal of Computational Intelligence. 3 (1), p 8-13. http://proceedings.spiiras.nw.ru/ ojs/index.php/sp/article/view/1134

6. Chernykh, YV (2003). "Methods of the analysis of time series connected with solar activity." SPIRAS Proceedings. 3 (1), p. 126-136.

7. Zurbenko, I. G \& Potrzeba, A.L (2013) "Tides in the Atmosphere." Air Quality, Atmos, \& Health, 6 (1): 39-46. Springer, DOI 10.1007/s11869-011-0143-6. http://www.springerlink.com/content/e124604331626295

8. Zurbenko, I.G \& Potrzeba, A.L (2009) "Tidal Waves in Atmosphere and Their Effects." Acta Geophysica, Springer, Vol 58 (2): 356-373. DOI 10.2478/s11600-009-0049-y

9. Strugarek A, Beaudoin P, Charbonneau P, Brun, A.S, do Nascimento Jr, J.D. (2017). "Reconciling solar and stellar magnetic cycles with nonlinear dynamo simulations." Science Vol. 357 (6347) p. 185-187. DOI: 10.1126/science.aal3999

10. Valachovic, E. \& Zurbenko I (2014). "Skin Cancer, Irradiation, and Sunspots: The Solar Cycle Effect." Biomedical Research International. Vol 2014. http://dx.doi.org/10.1155/2014/538574

11. WDC-SILSO, Royal Observatory of Belgium, Brussels. Sunspot Numbers: http://www.sidc.be/silso/datafiles

12. Frohlich C, Crommelynck D, Wehrli C, Anklin M, Dewitte S, Fichot A, Finsterle W, Jiménez, A. Chevalier A, and Roth, HJ. (1997). "In-flight performances of VIRGO solar irradiance instruments on SOHO." Sol. Phys., 175:267â€"286. doi:10.1023/A:1004929108864

13. Research Data Archive/Computational and Information Systems Laboratory/National Center for Atmospheric Research/University Corporation for Atmospheric Research, et al. 1984, updated monthly. International Comprehensive Ocean-Atmosphere Data Set (ICOADS) Release 2.5, Monthly Summaries. Research Data Archive at the National Center for Atmospheric Research, Computational and Information Systems Laboratory. https://doi.org/10.5065/D6CF9N3F. Accessed $\dagger 01$ Mar 2018.

14. Stephenson, FR \& Clark, DH (1978). Applications of Early Astronomical Records. Monographs on Astronomical Subjects: 4. Oxford University Press, New York.

15. Yang, W. \& Zurbenko I (2012), KZFT package version 0.17, R-software first published version (19-Sept-2007) and latest published version (2012), Package Sources https://cran.r-project.org/web/packages/kzft/kzft.pdf

16. DiRienzo G, Zurbenko I. (1999). "Semi-adaptive nonparametric spectral estimation." Journal of Computational Graphical Statistics. 8:41-59.

17. Zurbenko, I.G (1986). The Spectral Analysis of Time Series. North-Holland Series in Statistics in Probability.

18. Close B \& Zurbenko I (2013), KZFT in KZA package, R-software first published version kza_0.4 (27 Apr 2004) and latest published version (2013), Package Sources. http://cran.r-project.org/web/packages/kza/index.html

19. Yang, W \& Zurbenko, I.G (2010). "Kolmogorov-Zurbenko filters." Wiley interdisciplinary revives in computational statistics. WIREs in Comput Stat 2: 340-351. http://wires.wiley.com/WileyCDA/WiresArticle/ wisID-WICS71.html

20. Zurbenko, I.G. \& Smith, D. (2017). "Kolmogorov-Zurbenko filters in spatiotemporal analysis." WIREs Comput Stat, e1419. DOI: 10.1002/wics.1419

21. Valachovic, E. \& Zurbenko I (2017). "Separation of Spatio-Temporal Frequencies." Quest Journals: Journal of Research in Applied Mathematics. Vol 3 (7), p. 29-37.

22. Zurbenko I.G. \& Cyr, D.D (2011). "Climate fluctuations in time and space." Climate Research. Vol (46) p. 6776. DOI $10.3354 / \operatorname{cr} 00956$.

23. Tsakiri K, Zurbenko I (2010). "Prediction of Ozone Concentrations using Atmospheric Variables." Journal Air Quality, Atmosphere $\&$ Health. Vol 4 (2):111-120. DOI: 10.1007/s11869-010-0084-5

24. Zurbenko I, Sowizral, M (1999). "Resolution of the destructive effect on noise on linear regression of two time series." Far East Journal of Theoretical Statistics. Vol 3 (1): 139-157.

25. Zurbenko, I.G. \& Sun, M (2014). "Periods of High Risk in Tornado Outbreaks in Central USA." Advances in Research, 2 (8) http://www.sciencedomain.org/issue.php?iid=512\&id=31

26. Zurbenko, I.G. \& Sun, M (2015). "Associations of Jet Streams with Tornado Outbreaks in the North America." Atmospheric and Climate Sciences, Vol. 5 (3), p. 336-344. http://dx.doi.org/10.4236/acs.2015.53026

27. Zurbenko, I.G. \& Sun, M (2016). "Jet Stream as a major factor of tornados in USA." Atmospheric and Climate Sciences, Vol. 6 (2), p. 236 - 253. DOI: 10.4236/acs.2016.62020 http://www.scirp.org/journal/ACS/.

28. Zurbenko, I. G \& Potrzeba, AL (2013a). "Periods of Excess Energy in Extreme Weather Events." Journal of Climatology, Vol. 2013. Article ID 410898. http://dx.doi.org/10.1155/2013/410898 